\title{
Efeitos da Dexmedetomidina sobre o Sistema Renal e sobre a Concentração Plasmática do Hormônio Antidiurético. Estudo Experimental em Cães *
}

\section{Effects of Dexmedetomidine on Renal System and on Vasopressin Plasma Levels. Experimental Study in Dogs}

Nivaldo Ribeiro Villela ${ }^{1}$; Paulo do Nascimento Júnior, TSA ${ }^{2}$; Lídia Raquel de Carvalho ${ }^{3}$; Andrey Teixeira ${ }^{4}$

\section{RESUMO}

Villela NR, Nascimento Jr P, Carvalho LR, Teixeira A - Efeitos da Dexmedetomidina sobre o Sistema Renal e sobre a Concentração Plasmática do Hormônio Antidiurético. Estudo Experimental em Cães

JUSTIFICATIVA E OBJETIVOS: A insuficiência renal aguda peri-operatória é responsável por elevada taxa de morbidade e mortalidade. Os fármacos $\alpha_{2}$-agonistas aumentam o débito urinário e promovem boa estabilidade hemodinâmica nesse período. O objetivo desta pesquisa foi estudar os efeitos renais e sobre a concentração plasmática do hormônio antidiurético (HAD) provocados pela dexmedetomidina no cão anestesiado.

MÉTODO: Trinta e seis cães adultos, anestesiados com propofol, fentanil e isoflurano, foram divididos aleatoriamente em três grupos que receberam, de modo encoberto: $\mathrm{G} 1$ - injeção de $20 \mathrm{~mL}$ de solução de cloreto de sódio a 0,9\%, em 10 minutos, seguida de injeção de $20 \mathrm{~mL}$ da mesma solução em uma hora; G2 - injeção de $20 \mathrm{~mL}$ de solução de cloreto de sódio a $0,9 \%$ contendo dexmedetomidina $\left(1 \mu \mathrm{g} \cdot \mathrm{kg}^{-1}\right)$, em 10 minutos, seguida de injeção de $20 \mathrm{~mL}$ da mesma solução, com a mesma dose de dexmedetomidina $\left(1 \mu \mathrm{g} \cdot \mathrm{kg}^{-1}\right)$, em uma hora e G3 - injeção de $20 \mathrm{~mL}$ de solução de cloreto de sódio a $0,9 \%$ contendo dexmedetomidina $\left(2 \mu \mathrm{g} \cdot \mathrm{kg}^{-1}\right)$ em 10 minutos, seguida de injeção de $20 \mathrm{~mL}$ da mesma solução, com a mesma dose de dexmedetomidina $\left(2 \mu \mathrm{g} \cdot \mathrm{kg}^{-1}\right)$, em uma hora. As variáveis renais, hemodinâmicas e a concentração plasmática do HAD foram estudadas em quatro momentos: M1 (controle) - imediatamente após o período de estabilização; M2 após a injeção inicial de $20 \mathrm{~mL}$ da solução em estudo, em 10 minutos, coincidindo com o início da injeção da mesma solução, em uma hora; M3 - 30 minutos após M2 e M4 - 30 minutos após M3.

RESULTADOS: A dexmedetomidina reduziu a freqüência cardíaca e promoveu estabilidade hemodinâmica, mantendo constante o débito cardíaco. Houve elevação do débito urinário no G2 e G3, em

\footnotetext{
* Recebido do (Received from) Departamento de Anestesiologia da Faculdade de Medicina de Botucatu (FMB - UNESP), Botucatu, SP; Financiado pela Fundação de Amparo à Pesquisa do Estado de São Paulo FAPESP, Processo Auxílio Pesquisa n 03/01325-9

1. Doutor em Anestesiologia pela FMB - UNESP

2. Professor Adjunto Livre-Docente do Departamento de Anestesiologia da FMB - UNESP

3. Professora Assistente Doutora do Departamento de Bioestatística do Instituto de Biociências de Botucatu, UNESP

4. Doutorando em Reprodução Animal da Faculdade de Veterinária de Botucatu, UNESP
}

Apresentado (Submitted) em 09 de novembro de 2004

Aceito (Accepted) para publicação em 08 de março de 2005

Endereço para correspondência (Correspondence to)

Dr. Nivaldo Ribeiro Villela

Rua Nascimento Silva, 76/204 - Ipanema

22421-020 Rio de Janeiro, RJ

E-mail: nivaldovillela@terra.com.br

(c) Sociedade Brasileira de Anestesiologia, 2005 comparação com o G1. A osmolalidade urinária no G2 e G3 foi menor no $M 3$ e M4 em relação ao M1 e M2. A depuração de água livre aumentou no G3. A concentração plasmática do HAD diminuiu no $G 3$, apresentando valores mais baixos que os observados no G1 e $\mathrm{G} 2$ em M2 e M4.

CONCLUSÕES: Os cães anestesiados com baixas doses de dexmedetomidina promovem diurese hídrica por inibir a secreção do hormônio antidiurético, havendo potencial para proteção renal em eventos isquêmicos.

Unitermos: ANIMAIS: cães; DROGAS: $\alpha_{2}$-agonista, dexmedetomidina; SISTEMA RENAL: hormônio antidiurético

\section{SUMMARY}

Villela NR, Nascimento Jr P, Carvalho LR, Teixeira A - Effects of Dexmedetomidine on Renal System and on Vasopressin Plasma Levels. Experimental Study in Dogs

BACKGROUND AND OBJECTIVES: Perioperative acute renal failure increases morbidity and mortality. Alpha ${ }_{2}$-adrenergic agonists used in anesthesia increase urinary output and maintain cardiovascular stability. This study aimed at investigating the effects of dexmedetomidine on renal system and on vasopressin plasma levels in anesthetized dogs.

METHODS: This study involved 36 adult mixed-breed dogs anesthetized with propofol, fentanyl, and isoflurane, which were randomly distributed in three groups receiving: $\mathrm{G} 1-20 \mathrm{~mL}$ of $0.9 \%$ saline in 10 minutes, followed by $20 \mathrm{~mL}$ of the same solution in one hour; $\mathrm{G} 2-20 \mathrm{~mL}$ of $0.9 \%$ saline with dexmedetomidine $\left(1 \mu \mathrm{g} . \mathrm{kg}^{-1}\right)$ in 10 minutes, followed by $20 \mathrm{~mL}$ of the same solution with the same dexmedetomidine dose $\left(1 \mu \mathrm{g} \cdot \mathrm{kg}^{-1}\right)$ in one hour; and $\mathrm{G} 3-20 \mathrm{~mL}$ of $0.9 \%$ saline with dexmedetomidine $\left(2 \mu \mathrm{g} . \mathrm{kg}^{-1}\right)$ in 10 minutes, followed by $20 \mathrm{~mL}$ of the same solution with the same dexmedetomidine dose $\left(2 \mu \mathrm{g} \cdot \mathrm{kg}^{-1}\right)$ in one hour. Renal and hemodynamic variables and vasopressin plasma levels were studied in four periods: M1 (control) - immediately after the stabilization period; M2 - after initial injection of the solution being studied, coinciding with the beginning of continuous injection of the same solution; M3 - 30 minutes after M2; and M4 - 30 minutes after M3.

RESULTS: Dexmedetomidine has decreased heart rate and maintained adequate cardiovascular stability, as cardiac output was kept constant. Urinary output was increased for $\mathrm{G} 2$ and $\mathrm{G} 3$ as compared to $\mathrm{G} 1$. For $\mathrm{G} 2$ and $\mathrm{G} 3$, urinary osmolality has decreased in $\mathrm{M} 3$ and $\mathrm{M} 4$. For G3, values of free water clearance were increased throughout the experiment. Vasopressin plasma levels have decreased for G3, resulting in lower values as compared to $\mathrm{G} 1$ and $\mathrm{G} 2$ in M2 and M4.

CONCLUSIONS: In anesthetized dogs, low doses of dexmedetomidine inhibit vasopressin secretion, causing aqueous diuresis. These actions might protect kidneys during ischemic events.

Key Words: ANIMALS: dogs, DRUGS: $\alpha_{2}$-agonist, dexmedetomidine; RENAL SYSTEM: vasopressin 


\section{INTRODUÇÃO}

$\mathrm{N}$ as últimas décadas têm sido observados avanços nos cuidados médicos peri-operatórios, sem, contudo, que se verifique redução significativa da incidência de insuficiência renal aguda nesse período ${ }^{1-3}$. As elevadas taxas de morbidade e mortalidade associadas à insuficiência renal aguda peri-operatória justificam os esforços e gastos despendidos em pesquisas, na tentativa de identificar mecanismos de preveni-la ${ }^{4}$.

As alterações hemodinâmicas e hormonais decorrentes da resposta endócrino-metabólica ao trauma anestésico-cirúrgico desempenham importante papel na precipitação da insuficiência renal aguda ${ }^{5}$.

A dexmedetomidina reduz a concentração plasmática das catecolaminas ${ }^{6,7}$, mantém boa estabilidade hemodinâmica ${ }^{8,9}$ e aumenta o débito urinário ${ }^{10}$, quando empregada durante cirurgia, podendo assim, reduzir as alterações renais promovidas pela resposta endócrino-metabólica ao trauma anestésico-cirúrgico.

Na literatura, há escassez de trabalhos experimentais com a dexmedetomidina em condições e doses semelhantes àquelas praticadas nas salas de operações. Assim, o objetivo deste trabalho foi estudar os efeitos da dexmedetomidina sobre o sistema renal e sobre a concentração plasmática do hormônio antidiurético (HAD), em doses semelhantes às utilizadas na prática clínica em Anestesiologia.

\section{MÉTODO}

Esta pesquisa foi aprovada pela Comissão de Ética em Experimentação Animal da Faculdade de Medicina de Botucatu, UNESP. Foram utilizados 36 cães adultos de ambos os sexos, sem raça definida, com peso entre 18 e $30 \mathrm{~kg}$.

Após jejum alimentar de 12 horas, com livre acesso à água, os animais foram anestesiados com propofol $\left(6 \mathrm{mg} \cdot \mathrm{kg}^{-1}\right) \mathrm{e}$ fentanil $\left(5 \mu \mathrm{g} \cdot \mathrm{kg}^{-1}\right)$. Após a intubação traqueal, os pulmões foram ventilados mecanicamente com oxigênio $\left(0,8 \mathrm{~L} \cdot \mathrm{min}^{-1}\right)$ e ar comprimido (1,2 L. $\left.\mathrm{min}^{-1}\right)$, com volume corrente de 20 $\mathrm{mL} . \mathrm{kg}^{-1}$ e freqüência respiratória de 12 a 16 movimentos por minuto, com o objetivo de manter a pressão expiratória final de $\mathrm{CO}_{2}\left(\mathrm{P}_{\mathrm{ET}} \mathrm{CO}_{2}\right)$ em 35 a $45 \mathrm{mmHg}$, e iniciou-se administração de isoflurano em concentração expirada de 1,7 CAM. Realizou-se dissecção e cateterismo da veia femoral direita para início da hidratação com solução de Ringer com lactato $\left(18 \mathrm{~mL} \cdot \mathrm{kg}^{-1} \cdot \mathrm{h}^{-1}\right)$ e administrou-se o brometo de rocurônio $(0,6$ $\mathrm{mg} \cdot \mathrm{kg}^{-1}$ e injeção contínua de $10 \mu \mathrm{g} \cdot \mathrm{kg}^{-1} \cdot \mathrm{min}^{-1}$ ).

A seguir, foram dissecadas a artéria femoral esquerda, para medida contínua da pressão arterial média (PAM), a veia femoral esquerda, para coleta de sangue, e a veia jugular externa direita, por onde foi passado o introdutor e o cateter de Swan-Ganz 7F até a artéria pulmonar, para medida do débito cardíaco por termodiluição e da pressão média do átrio direito (PAD). A manutenção da temperatura corporal habitual para cães $\left(39^{\circ} \mathrm{C}\right)$ foi feita através de insuflação de ar aquecido $\left(38\right.$ a $\left.42^{\circ} \mathrm{C}\right)$ na superfície ventral e aquecimento das soluções injetadas.
Após o término do preparo cirúrgico, as feridas cirúrgicas foram infiltradas com ropivacaína a $0,2 \%$, a concentração expirada do isoflurano foi reduzida para 0,6 CAM e injetou-se o prime das soluções de creatinina $\left(30 \mathrm{mg} \cdot \mathrm{kg}^{-1}\right)$ e para-aminohipurato de sódio (PAH) (4 mg. $\left.\mathrm{kg}^{-1}\right)$ para estudo da função renal. A solução de Ringer com lactato foi substituída por solução de creatinina a $0,6 \%$ e de PAH a $0,24 \%$ em Ringer com lactato e administrada em volume de $6 \mathrm{~mL} \cdot \mathrm{kg}^{-1} \cdot \mathrm{h}^{-1}$. Procedeu-se o cateterismo vesical e iniciou-se período de estabilização de 30 minutos.

Após o momento controle, os animais foram distribuídos de forma aleatória, por sorteio, e encoberta em três grupos com 12 cães em cada grupo.

- $\mathrm{G} 1$ ( $\mathrm{n}=12)$ : injeção de $20 \mathrm{~mL}$ de solução de cloreto de sódio a $0,9 \%$, em 10 minutos, seguida de injeção de $20 \mathrm{~mL}$ da mesma solução em uma hora.

- $\mathrm{G} 2(\mathrm{n}=12)$ : injeção de $20 \mathrm{~mL}$ de solução de cloreto de sódio a $0,9 \%$ contendo dexmedetomidina $\left(1 \mu \mathrm{g} \cdot \mathrm{kg}^{-1}\right)$, em 10 minutos, seguida de injeção de $20 \mathrm{~mL}$ da mesma solução, com a mesma dose de dexmedetomidina (1 $\left.\mu \mathrm{g} \cdot \mathrm{kg}^{-1}\right)$, em uma hora.

- $\mathrm{G} 3(\mathrm{n}=12)$ : injeção de $20 \mathrm{~mL}$ de solução de cloreto de sódio a $0,9 \%$ contendo dexmedetomidina $\left(2 \mu \mathrm{g} \cdot \mathrm{kg}^{-1}\right)$, em 10 minutos, seguida de injeção de $20 \mathrm{~mL}$ da mesma solução, com a mesma dose de dexmedetomidina (2 $\mu \mathrm{g} \cdot \mathrm{kg}^{-1}$ ), em uma hora.

Foram estudados os seguintes parâmetros para controle da homogeneidade do estudo - comprimento, peso, área de superfície corporal (ASC) e sexo; atributos hemodinâmicos freqüência cardíaca ( $F C)$, pressão arterial média (PAM), pressão média do átrio direito (PAD), índice cardíaco (IC) e o índice da resistência vascular sistêmica (IRVS); atributos renais e sangüíneos - hematócrito $(\mathrm{Ht})$, osmolalidade plasmática (OsmP) débito urinário (DU), ritmo de filtração glomerular (RFG), medido pela depuração (Dp) de creatinina (DpCr), fluxo sangüíneo renal (FSR = DpPAH/1-Ht), resistência vascular renal $(\mathrm{RVR}=\mathrm{PAM} / \mathrm{FSR})$, osmolalidade urinária (OsmU), depuração osmolar (DpOsm = OsmU.DU/OsmP), depuração de água livre ( $\left.\mathrm{DpH}_{2} \mathrm{O}=\mathrm{DU}-\mathrm{DpOsm}\right)$, fração de filtração ( $F F=D p C r / D p P A H)$, excreção fracionária de sódio $(\mathrm{EFNa}=\mathrm{DpNa} \cdot 100 / \mathrm{DpCr})$, excreção fracionária de potássio (EFK = DpK.100/DpCr) e a concentração plasmática do hormônio antidiurético (HAD).

Os parâmetros foram estudados em 4 momentos. Cada momento teve duração de 15 minutos, sendo que a coleta de sangue e a medida das variáveis hemodinâmicas foram realizadas na metade desse intervalo. No início e final de cada momento, a bexiga foi esvaziada e a urina coletada. Os momentos estudados foram:

M1 (controle): imediatamente após o período de estabilização de 30 minutos.

M2: após a injeção inicial, em 10 minutos, de $20 \mathrm{~mL}$ da solução em estudo, coincidindo com o início da injeção contínua da solução em estudo, em uma hora. 
M3: 30 minutos após o término de M2, coincidindo o término deste momento com o término da injeção da solução em estudo.

M4: 30 minutos após o término de M3.

Para a dosagem plasmática do hormônio antidiurético (HAD) foram coletados $5 \mathrm{~mL}$ de sangue venoso em tubos heparinizados que foram centrifugados a $4{ }^{\circ} \mathrm{C}$ para separação do plasma. Foi utilizada a técnica de radioimunoensaio ( $k i t$ DSL-1800 Arginine Vasopressin Radioimmunoassay, Texas) e a leitura foi realizada no aparelho Perkim-Elmer Cobra II Gamma Counter, modelo E5005 (USA). A concentração plasmática do HAD foi expressa em picogramas por $\mathrm{mL}$ de plasma.

Para as variáveis que apresentaram distribuição normal e homogeneidade de variâncias, foi utilizada a análise de perfil, seguida do método de Tukey para comparações múltiplas. Para as que não apresentaram distribuição normal ou homogeneidade de variâncias, foi utilizado o teste de Friedman para comparações dos momentos e o teste de Kruskal-Wallis para comparação dos grupos, seguidos do teste para comparações múltiplas. Os dados demográficos foram verificados pelo método de Análise de Variância. Com relação ao sexo, empregou-se o teste Exato de Fisher para análise de freqüência. O nível de significância utilizado foi de 5\%.

\section{RESULTADOS}

Houve homogeneidade entre os grupos em relação ao peso, comprimento, ASC e distribuição de machos e fêmeas dos animais $(p>0,05)$ (Tabela I).

Tabela I - Peso, Comprimento, Área de Superfície Corporal (ASC) e Distribuição de Machos e Fêmeas em cada um dos Grupos Estudados

\begin{tabular}{lcccc}
\hline Parâmetros & $\begin{array}{c}\mathrm{G} 1 \\
(\mathrm{n}=12)\end{array}$ & $\begin{array}{c}\mathrm{G} 2 \\
(\mathrm{n}=12)\end{array}$ & $\begin{array}{c}\mathrm{G} 3 \\
(\mathrm{n}=12)\end{array}$ & Estatística \\
\hline Peso $(\mathrm{kg})^{*}$ & $24,3 \pm 4,2$ & $21,9 \pm 3,9$ & $23,9 \pm 3,7$ & $\mathrm{p}>0,05$ \\
Comprimento $(\mathrm{cm}){ }^{*}$ & $114,7 \pm 6,9$ & $107,3 \pm 8,0$ & $114,8 \pm 10,1$ & $\mathrm{p}>0,05$ \\
ASC $\left(\mathrm{m}^{2}\right)^{*}$ & $0,90 \pm 0,09$ & $0,85 \pm 0,09$ & $0,86 \pm 0,11$ & $\mathrm{p}>0,05$ \\
Sexo & & & & \\
\multicolumn{1}{c}{ Machos } & 9 & 10 & 12 & \\
Fêmeas & 3 & 2 & 0 & $\mathrm{p}>0,05$ \\
\hline
\end{tabular}

* Valores expressos pela Média \pm DP

As variáveis PAM e PAD não apresentaram diferença significativa entre os grupos e nos diferentes momentos de cada grupo $(p>0,05)$ (Tabela II).

Tabela II - Valores da Freqüência Cardíaca (FC), Pressão Arterial Média (PAM), Pressão Média do Átrio Direito (PAD), do Índice Cardíaco (IC) e do Índice da Resistência Vascular Sistêmica (IRVS) nos Momentos e Grupos Estudados

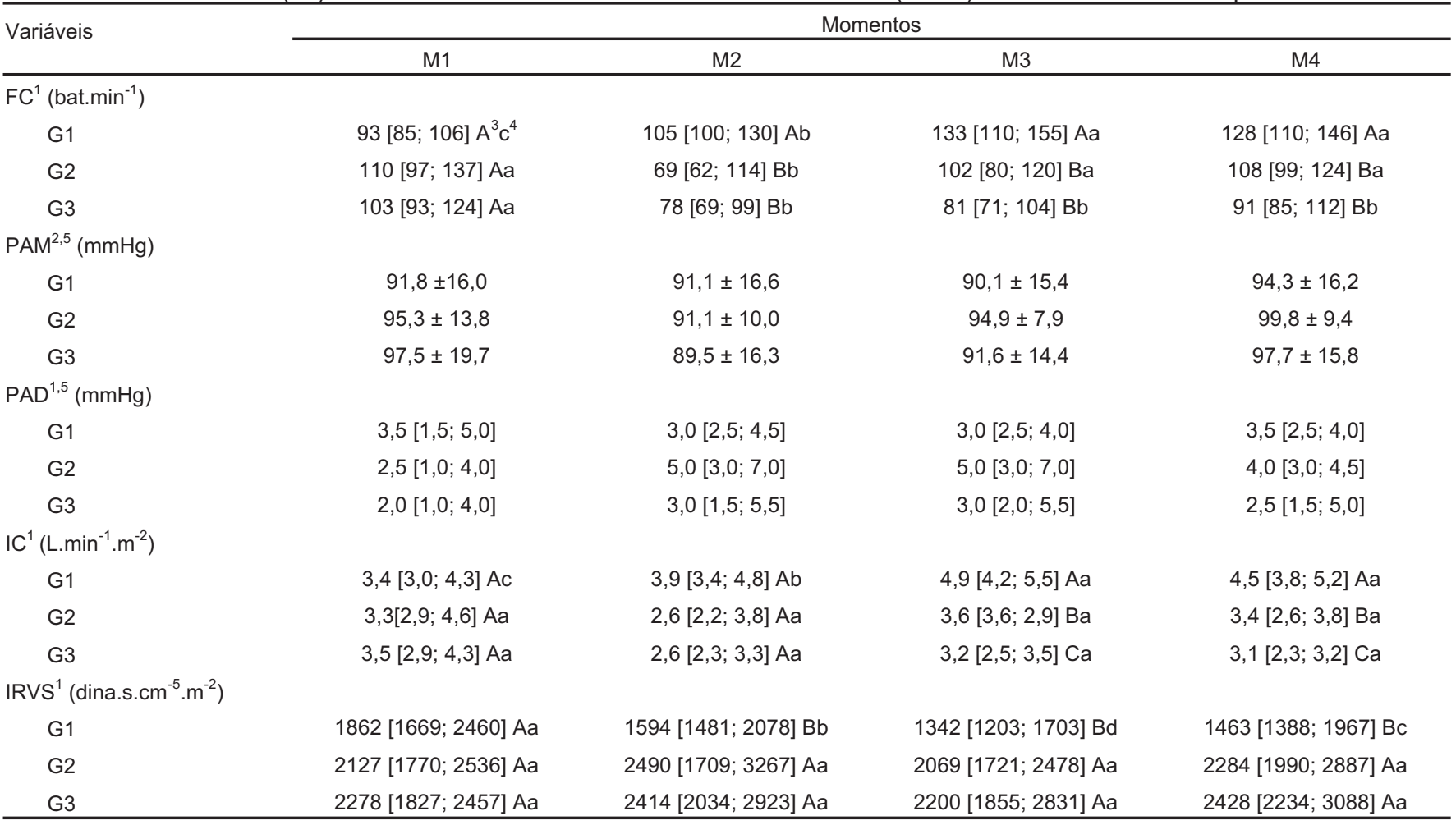

${ }^{1}$ Mediana, $1^{\circ}$ e $3^{\circ}$ quartis; ${ }^{2}$ Média \pm DP; ${ }^{3}$ Grupos dentro de cada momento, seguidos de mesma letra maiúscula, não diferem estatisticamente ( $p>0,05$ ); ${ }^{4}$ Momentos dentro de cada grupo, seguidos de mesma letra minúscula, não diferem estatisticamente $(p>0,05) ;{ }^{5} p>0,05$ (entre grupos, dentro de cada momento e entre momentos, dentro de cada grupo). ${ }^{3,4}$ Observação quanto aos valores: $A>B>C ; a>b>c>d$ 
AFC foi menor no G2 e G3, em relação ao G1, no M2, M3 e M4 $(p<0,05)$. Nos grupos, observou-se que no G2 a FC diminuiu no M2 $(p<0,05)$, retornando aos valores do momento controle no M3 e M4. No G3, a FC diminuiu no M2 e manteve-se abaixo dos valores do momento controle no M3 e M4 $(p<0,05)$. O G1 apresentou aumento progressivo da FC $(p<0,05)$ (Tabela II).

O IC, no M3 e M4, foi menor no G2 e G3 em relação ao G1 e no $\mathrm{G} 3$ em relação ao $\mathrm{G} 2(\mathrm{p}<0,05)$. No $\mathrm{G} 1$, houve aumento progressivo do IC ao longo dos momentos $(p<0,05)$ (Tabela II).

O IRVS foi menor no G1 no M2, M3 e M4 em relação ao G2 e G3 ( $p<0,05)$. No G1, houve redução do IRVS nos momentos $M 2, M 3$ e M4 em relação ao M1 $(p<0,05)$ (Tabela II). ODU foi menor no G1, no M4 ( $p<0,05)$. No G3, o DU foi menor no $M 2$ em relação ao M3 $(p<0,05)$ (Figura 1).

AFF foi menor no $G 2$ em relação ao $G 1$, no $M 1(p<0,05)$. No $\mathrm{G} 2$, foi maior no M4 em relação ao M1 ( $p<0,05)$ (Tabela III). A OsmU não apresentou diferença significativa entre os grupos. No G2 e G3, foi menorno M3 e M4 ( $p<0,05)$ (Tabela IV). $\mathrm{ADpH} \mathrm{H}_{2} \mathrm{O}$ não apresentou diferença significativa entre os grupos. No G3, foi maior no M2, M3 e M4 em relação ao M1 ( $p$ < 0,05) (Figura 2).

A concentração plasmática do HAD foi menor no G3 em relação aos $\mathrm{G} 1$ e G2 e no $\mathrm{G} 2$ em relação ao $\mathrm{G} 1$, no M2 e M4. ( $p$ < $0,05)$. No G3, foi maior no M1 $(p<0,05)$ (Figura 3).

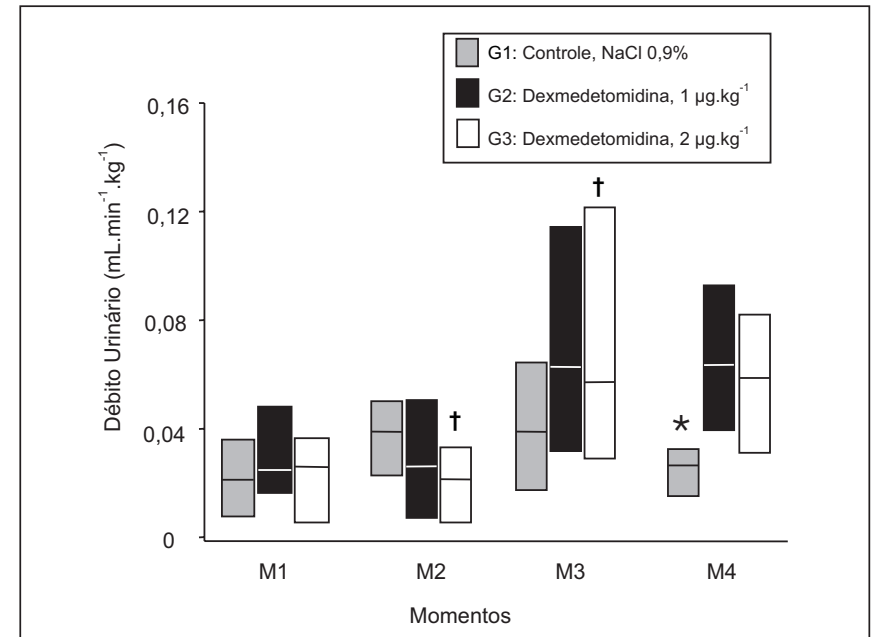

Figura 1 - Débito Urinário. Mediana, $1^{\circ}$ e $3^{\circ}$ Quartis dos Valores Obtidos nos Grupos e Momentos Estudados

* $M 4: G 1<(G 2=G 3)(p<0,05)$

+ G3: $M 2<M 3(p<0,05)$

As variáveis FSR, RFG, RVR (Tabela III), Ht, OsmP, DpOsm, EFNa e EFK (Tabela IV) não apresentaram diferença significativa entre os grupos e nos diferentes momentos de cada grupo $(p>0,05)$.

Tabela III - Valores do Fluxo Sangüíneo Renal (FSR), Ritmo de Filtração Glomerular (RFG), da Resistência Vascular Renal (RVR) e da Fração de Filtração (FF) nos Momentos e Grupos Estudados

\begin{tabular}{|c|c|c|c|c|}
\hline \multirow[t]{2}{*}{ Variáveis } & \multicolumn{4}{|c|}{ Momentos } \\
\hline & M1 & M2 & M3 & M4 \\
\hline \multicolumn{5}{|c|}{$\mathrm{FSR}^{2}\left(\mathrm{~mL} \cdot \mathrm{min}^{-1} \cdot \mathrm{kg}^{-1}\right)$} \\
\hline G1 & $16,1 \pm 5,2$ & $20,9 \pm 7,3$ & $18,8 \pm 5,4$ & $19,1 \pm 6,5$ \\
\hline $\mathrm{G} 2$ & $24,5 \pm 8,3$ & $20,0 \pm 6,4$ & $20,1 \pm 4,4$ & $21,1 \pm 5,6$ \\
\hline G3 & $19,1 \pm 10,0$ & $17,9 \pm 10,3$ & $19,5 \pm 11,4$ & $17,8 \pm 9,5$ \\
\hline \multicolumn{5}{|c|}{$\mathrm{RFG}^{1,5}\left(\mathrm{~mL} \cdot \mathrm{min}^{-1} \cdot \mathrm{kg}^{-1}\right)$} \\
\hline G1 & $3,4[2,5 ; 4,3]$ & $3,9[3,1 ; 5,0]$ & $3,7[3,2 ; 4,7]$ & $3,5[3,0 ; 4,0]$ \\
\hline G2 & $3,7[2,8 ; 4,4]$ & $4,1[3,8 ; 4,6]$ & $3,9[3,2 ; 4,4]$ & $4,0[3,7 ; 4,8]$ \\
\hline G3 & $3,4[2,6 ; 4,8]$ & $3,5[2,9 ; 4,8]$ & $3,7[3,4 ; 4,5]$ & $3,8[3,4 ; 4,3]$ \\
\hline \multicolumn{5}{|c|}{$\mathrm{RVR}^{1,5}\left(\mathrm{mmHg} \cdot \mathrm{mL}^{-1} \cdot \mathrm{min}^{-1}\right)$} \\
\hline G1 & $0,25[0,19 ; 0,32]$ & $0,19[0,14 ; 0,25]$ & $0,18[0,16 ; 0,24]$ & $0,20[0,16 ; 0,27]$ \\
\hline G2 & $0,17[0,15 ; 0,22]$ & $0,23[0,19 ; 0,26]$ & $0,24[0,18 ; 0,28]$ & $0,26[0,20 ; 0,30]$ \\
\hline G3 & $0,24[0,17 ; 0,32]$ & $0,24[0,14 ; 0,34]$ & $0,23[0,16 ; 0,30]$ & $0,26[0,18 ; 0,36]$ \\
\hline \multicolumn{5}{|l|}{$\mathrm{FF}^{2}$} \\
\hline G1 & $0,38 \pm 0,09$ a3A4 & $0,34 \pm 0,09 a A$ & $0,36 \pm 0,09 a A$ & $0,35 \pm 0,07 \mathrm{aA}$ \\
\hline $\mathrm{G} 2$ & $0,27 \pm 0,09 \mathrm{bB}$ & $0,33 \pm 0,06 a b A$ & $0,34 \pm 0,10 a b A$ & $0,35 \pm 0,07 \mathrm{aA}$ \\
\hline G3 & $0,37 \pm 0,09 a \mathrm{aB}$ & $0,38 \pm 0,09 a A$ & $0,36 \pm 0,09 a A$ & $0,41 \pm 0,07 \mathrm{aA}$ \\
\hline
\end{tabular}

${ }^{1}$ Mediana, $1^{\circ}$ e $3^{\circ}$ quartis; ${ }^{2}$ Média $\pm \mathrm{DP} ;{ }^{3} \mathrm{Grupos}$ dentro de cada momento, seguidos de mesma letra maiúscula, não diferem estatisticamente ( $\mathrm{p}>0,05$ ); ${ }^{4}$ Momentos dentro de cada grupo, seguidos de mesma letra minúscula, não diferem estatisticamente $(p>0,05) ;{ }^{5} p>0,05$ (entre grupos, dentro de cada momento e entre momentos, dentro de cada grupo); ${ }^{3,4}$ Observação quanto aos valores: $A>B ; a>b$ 
Tabela IV - Valores do Hematócrito $(\mathrm{Ht})$, da Osmolalidade Urinária (OsmU), Osmolalidade Plasmática (OsmP), Depuração Osmolar (DpOsm), Excreção Fracionária de Sódio (EFNa) e Excreção Fracionária de Potássio (EFK) nos Momentos e Grupos Estudados

\begin{tabular}{|c|c|c|c|c|}
\hline \multirow[t]{2}{*}{ Variáveis } & \multicolumn{4}{|c|}{ Momentos } \\
\hline & M1 & M2 & M3 & M4 \\
\hline \multicolumn{5}{|l|}{$\mathrm{Ht}^{2}(\%)$} \\
\hline $\mathrm{G} 1$ & $39,5[36,0 ; 43,0]$ & $40,0[36,5 ; 44,0]$ & $41,0[37,0 ; 43,5]$ & $41,0[37,0 ; 42,5]$ \\
\hline $\mathrm{G} 2$ & $41,0[38,5 ; 44,5]$ & $42,0[36,0 ; 46,0]$ & $41,5[39,0 ; 46,5]$ & $43,5[40,0 ; 46,0]$ \\
\hline G3 & $39,5[36,5 ; 42,0]$ & $41,0[35,5 ; 44,0]$ & $41,5[36,0 ; 44,0]$ & $41,5[36,0 ; 45,0]$ \\
\hline \multicolumn{5}{|c|}{ OsmU (mOsm.kg H $\mathrm{H}_{2} \mathrm{O}^{-1}$ ) } \\
\hline $\mathrm{G} 1$ & $992[679 ; 1369] a^{1}$ & $752[566 ; 1128]$ a & $641[435 ; 825]$ a & $665[404 ; 1026]$ a \\
\hline $\mathrm{G} 2$ & $754[561 ; 994] \mathrm{a}$ & 641 [536; 868] a & $358[302 ; 639] \mathrm{b}$ & $306[234 ; 655] \mathrm{b}$ \\
\hline G3 & $925[795 ; 1346]$ a & 795 [605; 986] a & $452[313 ; 558] \mathrm{b}$ & $344[282 ; 604] \mathrm{A}$ \\
\hline \multicolumn{5}{|c|}{ OsmP $P^{2}$ (mOsm.kg H $\mathrm{O}^{-1}$ ) } \\
\hline $\mathrm{G} 1$ & $288[278 ; 295]$ & $284[278 ; 288]$ & $288[287 ; 290]$ & $288[285 ; 293]$ \\
\hline $\mathrm{G} 2$ & $290[286 ; 295]$ & $293[284 ; 296]$ & $288[284 ; 292]$ & $290[283 ; 295]$ \\
\hline G3 & $281[252 ; 290]$ & $285[238 ; 295]$ & $286[269 ; 290]$ & $287[258 ; 293]$ \\
\hline \multicolumn{5}{|c|}{$\mathrm{DpOsm}^{2}\left(\mathrm{~mL} \cdot \mathrm{min}^{-1}\right)$} \\
\hline $\mathrm{G} 1$ & $1,9[1,2 ; 2,2]$ & $2,2[1,6 ; 2,7]$ & $1,9[1,6 ; 2,7]$ & $1,8[1,2 ; 2,0]$ \\
\hline $\mathrm{G} 2$ & $1,9[1,1 ; 2,9]$ & $1,4[1,2 ; 2,5]$ & $1,8[1,4 ; 3,2]$ & $1,7[1,2 ; 3,3]$ \\
\hline G3 & $2,1[1,6 ; 3,0]$ & $1,6[1,1 ; 1,9]$ & $1,9[1,5 ; 4,0]$ & $1,7[1,4 ; 2,3]$ \\
\hline \multicolumn{5}{|l|}{$\mathrm{EFNa}^{2}(\%)$} \\
\hline G1 & $0,38[0,23 ; 0,77]$ & $0,89[0,44 ; 1,07]$ & $0,94[0,25 ; 1,21]$ & $0,42[0,20 ; 0,64]$ \\
\hline $\mathrm{G} 2$ & $0,57[0,31 ; 1,08]$ & $0,55[0,37 ; 0,83]$ & $1,02[0,75 ; 1,28]$ & $0,59[0,40 ; 0,81]$ \\
\hline G3 & $0,40[0,23 ; 0,96]$ & $0,37[0,21 ; 1,05]$ & $0,68[0,42 ; 1,09]$ & $0,60[0,31 ; 0,99]$ \\
\hline \multicolumn{5}{|l|}{$\mathrm{EFK}^{2}(\%)$} \\
\hline $\mathrm{G} 1$ & $12,0[7,9 ; 24,7]$ & $16,7[12,1 ; 29,2]$ & $24,7[14,9 ; 31,2]$ & $21,0[12,4 ; 26,2]$ \\
\hline $\mathrm{G} 2$ & $21,8[11,1 ; 31,2]$ & $15,7[11,0 ; 26,8]$ & $31,1[23,4 ; 37,6]$ & $20,3[15,7 ; 28,8]$ \\
\hline G3 & $20,0[12,7 ; 29,2]$ & $15,0[5,7 ; 21,7]$ & $25,9[23,0 ; 28,0]$ & $20,2[14,3 ; 25,3]$ \\
\hline
\end{tabular}

Valores representados pela mediana, $1^{\circ}$ e $3^{\circ}$ quartis; ${ }^{1}$ Momentos dentro de cada grupo, seguidos de mesma letra minúscula, não diferem estatisticamente $(p>0,05) ;{ }^{2} p>0,05$ (entre grupos, dentro de cada momento e entre momentos, dentro de cada grupo); ${ }^{1}$ Observação quanto aos valores: a > b

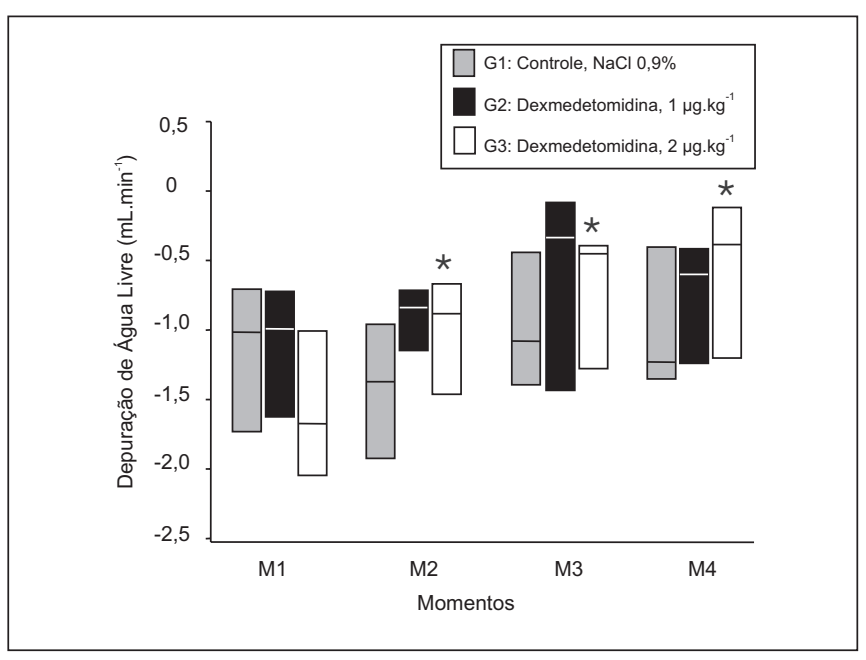

Figura 2 - Depuração de Água Livre. Mediana, $1^{\circ}$ e $3^{\circ}$ Quartis dos Valores Obtidos nos Grupos e Momentos Estudados * G3: M1< (M2=M3=M4) $(p<0,05)$

Revista Brasileira de Anestesiologia Vol. 55, № 4, Julho - Agosto, 2005

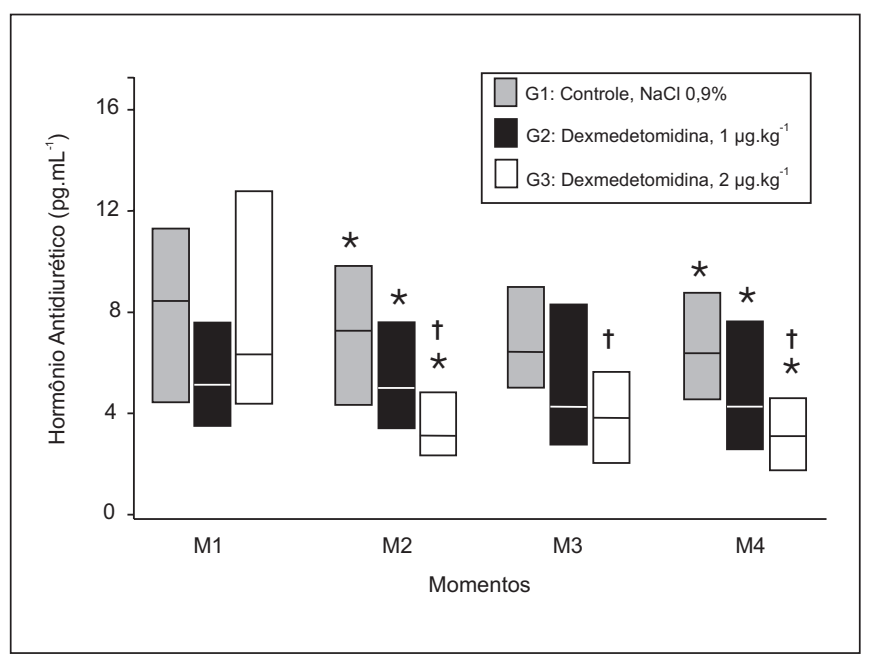

Figura 3 - Concentração Plasmática do Hormônio Antidiurético. Mediana, $1^{\circ}$ e $3^{\circ}$ Quartis dos Valores Obtidos nos Grupos e Momentos Estudados

* M2 e M4: G1>G2>G3 (p>0,05)

† G3: $M 1>(M 2=M 3=M 4)(p<0,05)$ 


\section{DISCUSSÃO}

A filtração glomerular é um mecanismo predominantemente hemodinâmico e a dinâmica intratubular renal sofre influências hemodinâmicas e hormonais. Alguns desses hormônios, como o HAD, sofrem interferências cardiovasculares no controle de sua secreção ${ }^{11}$. Observou-se neste experimento que as alterações urinárias promovidas pela dexmedetomidina não foram acompanhadas de modificações hemodinâmicas significativas. Assim como em outras pesquisas ${ }^{6-10,12}$, a dexmedetomidina diminuiu a FC de forma dose-dependente e manteve maior estabilidade hemodinâmica em relação ao grupo controle, uma vez que o IC manteve-se estável ao longo do experimento em ambos os grupos em que se empregou o fármaco.

Os agonistas $\alpha_{2}$-adrenérgicos aumentam o débito urinário 12-15. Esse aumento pode ser secundário às alterações hemodinâmicas ${ }^{16}$, à inibição da secreção do HAD ${ }^{17}$ ou a diminuição da sua ação tubular ${ }^{18}$.

Os receptores $\alpha_{2}$-adrenérgicos já foram identificados em várias áreas do rim de diferentes animais ${ }^{19-25}$. Em ratos, a ativação desses receptores promove inibição do AMPc intracelu$\operatorname{lar}^{26,27}$, sendo esse um dos mecanismos responsáveis pela inibição da ação do HAD no túbulo coletor. Em outros animais, como no cão, alguns pesquisadores não detectaram tal inibição do AMPc intracelular ${ }^{16}$, sugerindo outro mecanismo envolvido na inibição da absorção de água no túbulo coletor pelos agonistas $\alpha_{2}$-adrenérgicos. Reid e col. ${ }^{17}$ relataram que a clonidina em doses muito elevadas, bem acima das utilizadas na prática clínica, inibe a secreção do HAD, promovendo elevação da diurese.

Observou-se nesta pesquisa que a dexmedetomidina determinou aumento do débito urinário, promovendo, paralelamente, mínimas alterações hemodinâmicas. O aumento da diurese foi acompanhado de redução da osmolalidade urinária e aumento da depuração de água livre. Não foram observadas alterações nas variáveis RVR, FSR, RFG, DpOsm, EFNa e EFK, evidenciando que o aumento da diurese foi secundário ao comprometimento da absorção de água no túbulo coletor e não ao aumento da filtração glomerular. Provavelmente, essa diurese hídrica foi secundária à inibição da secreção do HAD.

Humphrey e col. ${ }^{28}$ associaram a inibição da secreção do HAD, após injeção de clonidina, ao aumento da PAM. Outros possíveis mecanismos seriam a supressão da secreção desse hormônio pelo aumento da pressão venosa central ${ }^{29}$ ou bloqueio direto das células neurossecretoras supra-ópticas ${ }^{30}$.

Neste experimento, a dexmedetomidina inibiu a secreção do HAD de forma dose-dependente sem que tenham ocorrido alterações significativas da PAM ou da PAD, sugerindo inibição central direta da droga. Em trabalho prévio, Kimura e col. ${ }^{31}$ observaram redução da concentração plasmática do HAD após injeção de pequena dose de clonidina no ventrículo lateral de cães, mostrando que os fármacos $\alpha_{2}$-agonistas podem promover bloqueio central da secreção desse hormônio.
Nesta pesquisa, durante o momento controle, observou-se que a concentração plasmática do HAD encontrava-se próxima dos valores fisiológicos para o cão $\left(0 \text { a } 5 \text { pg. } \mathrm{mL}^{-1}\right)^{32}$, sendo, provavelmente, o resultado da hidratação durante o preparo cirúrgico e da opção por uma técnica anestésica balanceada com o uso de opióide na indução ${ }^{33}$. Outros pesquisadores observaram valores sangüíneos bastante elevados do HAD após procedimentos anestésico-cirúrgicos em cães ${ }^{34}$. Após a administração de dexmedetomidina, em doses próximas daquelas utilizadas na prática clínica em Anestesiologia ${ }^{35}$, houve redução da concentração plasmática do HAD, elevação do débito urinário e produção de urina com baixa osmolalidade. Não foram observadas alterações hemodinâmicas que pudessem justificar a redução da secreção do HAD e a maior produção de urina.

Em trabalhos experimentais e clínicos, o bloqueio da resposta endócrino-metabólica ao trauma anestésico-cirúrgico associado aos efeitos renais promovidos pela clonidina foi capaz de prevenir ou diminuir as lesões renais secundárias à isquemia renal ${ }^{36,37}$.

Assim, concluiu-se que a administração de baixas doses de dexmedetomidina em cães resulta em produção de diurese hídrica, secundária à inibição central da secreção do HAD. Com base nessas ações e nas pesquisas realizadas com a clonidina, que resultaram em proteção renal frente à isquemia, sugere-se que a dexmedetomidina apresenta potencial papel na proteção renal em eventos isquêmicos. Modelos experimentais de isquemia renal, bem como estudos clínicos em pacientes susceptíveis à agressão isquêmica renal, são necessários para comprovar essa hipótese.

\section{Effects of Dexmedetomidine on Renal System and on Vasopressin Plasma Levels. Experimental Study in Dogs}

Nivaldo Ribeiro Villela, M.D.; Paulo do Nascimento Júnior, TSA, M.D., Lídia Raquel de Carvalho, M.D., Andrey Teixeira, M.D.

\section{INTRODUCTION}

Recent decades have witnessed perioperative care advances, however without significant decrease in the incidence of acute renal failure ${ }^{1-3}$. High morbidity and mortality rates associated to perioperative acute renal failure justify efforts and costs incurred in studies attempting to identify preventive mechanisms ${ }^{4}$.

Hemodynamic and hormonal changes promoted by endocrine-metabolic response to anesthetic-surgical trauma play an important role in triggering acute renal failure ${ }^{5}$.

Dexmedetomidine decreases catecholamine plasma levels 6,7 , maintains adequate hemodynamic stability ${ }^{8,9}$ and increases urinary output ${ }^{10}$ when administered during surgery, 
thus being able to decrease renal changes promoted by endocrine-metabolic response to anesthetic-surgical trauma. There are few experimental studies with dexmedetomidine in conditions and doses similar to those administered in operating rooms. So, this study aimed at evaluating the effects of dexmedetomidine on renal system and on vasopressin plasma levels, in doses similar to those used in clinical anesthesia.

\section{METHODS}

This study was approved by the Animal Experiment Ethics Committee, Faculdade de Medicina, Botucatu, UNESP and involved 36 adult mixed-breed dogs of both genders, weighing 18 to $30 \mathrm{~kg}$.

After 12-hour fasting with free access to water, anesthesia was induced with propofol $\left(6 \mathrm{mg} \cdot \mathrm{kg}^{-1}\right)$ and fentanyl (5 $\left.\mu \mathrm{g} . \mathrm{kg}^{-1}\right)$. After tracheal intubation, lungs were mechanically ventilated with oxygen $\left(0.8 \mathrm{~L} \cdot \mathrm{min}^{-1}\right)$ and compressed air (1.2 L. $\mathrm{min}^{-1}$ ), with tidal volume of $20 \mathrm{~mL} . \mathrm{kg}^{-1}$ and respiratory rate of 12 to 16 movements per minute, aiming at maintaining end tidal $\mathrm{CO}_{2}\left(\mathrm{P}_{\mathrm{ET}} \mathrm{CO}_{2}\right)$ between 35 and $45 \mathrm{mmHg}$, and isoflurane administration was started in expired concentration of 1.7 MAC. Right femoral vein was dissected and catheterized for hydration with lactated Ringer's solution $\left(18 \mathrm{~mL} \cdot \mathrm{kg}^{-1} \cdot \mathrm{h}^{-1}\right)$ followed by rocuronium injection $\left(0.6 \mathrm{mg} \cdot \mathrm{kg}^{-1}\right.$ and continuous injection of $\left.10 \mu \mathrm{g} \cdot \mathrm{kg}^{-1} \cdot \mathrm{min}^{-1}\right)$.

Next, left femoral artery was dissected for continuous mean blood pressure (MBP) monitoring, left femoral vein was dissected for blood collection, and right external jugular vein was dissected for introduction of $7 \mathrm{~F}$ Swan-Ganz catheter in the pulmonary artery to measure cardiac output by thermodilution and mean diastolic blood pressure (DBP). Normal body temperature for dogs $\left(39^{\circ} \mathrm{C}\right)$ was maintained with heated air blow ( 38 to $42^{\circ} \mathrm{C}$ ) on the ventral surface and warming of injected solutions.

After surgical preparation, surgical wounds were infiltrated with $0.2 \%$ ropivacaine, isoflurane expired concentration was decreased to 0.6 MAC and creatinine (30 mg. $\left.\mathrm{kg}^{-1}\right)$ and sodium para-aminohipurate $(\mathrm{PAH})\left(4 \mathrm{mg} \cdot \mathrm{kg}^{-1}\right)$ priming was injected to evaluate renal function. Lactated Ringer's solution was replaced by $0.6 \%$ creatinine and $6 \mathrm{~mL} \cdot \mathrm{kg}^{-1} \cdot \mathrm{h}^{-1}$ of $0.24 \%$ $\mathrm{PAH}$ solution in lactated Ringer's and was administered. Vesical catheter was inserted and a 30-minute stabilization period was started.

After the control moment, animals were randomly and blindly distributed in three groups of 12 dogs:

- $\mathrm{G} 1$ ( $\mathrm{n}=12): 20 \mathrm{~mL}$ of $0.9 \%$ saline in 10 minutes, followed by $20 \mathrm{~mL}$ of the same solution in one hour

- $\mathrm{G} 2(\mathrm{n}=12): 20 \mathrm{~mL}$ of $0.9 \%$ saline with dexmedetomidine $\left(1 \mu \mathrm{g} . \mathrm{kg}^{-1}\right)$ in 10 minutes, followed by $20 \mathrm{~mL}$ of the same solution with the same dexmedetomidine dose (1 $\left.\mu \mathrm{g} \cdot \mathrm{kg}^{-1}\right)$ in one hour

- $\mathrm{G} 3(\mathrm{n}=12): 20 \mathrm{~mL}$ of $0.9 \%$ saline with dexmedetomidine $\left(2 \mu \mathrm{g} . \mathrm{kg}^{-1}\right)$ in 10 minutes, followed by $20 \mathrm{~mL}$ of the same solution with the same dexmedetomidine dose (2 $\mu \mathrm{g} \cdot \mathrm{kg}^{-1}$ ) in one hour.

To control study homogeneity, the following parameters were evaluated: length, weight, body surface area (BSA) and gender. Evaluated hemodynamic parameters were: heart rate (HR), mean blood pressure (MBP), mean diastolic pressure (MDP), cardiac index $(\mathrm{Cl})$ and systemic vascular resistance (SVR); blood and renal parameters were: hematocrit $(\mathrm{Ht})$, plasma osmolality (OsmP), urinary output (UO), glomerular filtration rate (GFR) measured by creatinine clearance (CLCr), renal blood flow (RBF - CLPAH/1-Ht), renal vascular resistance (RVR - MBP/RBF), urinary osmolality (OsmU), osmolar clearance (CLOsm = OsmU.UO/OsmP), free water clearance $\left(\mathrm{CLH}_{2} \mathrm{O}=\mathrm{UO}-\mathrm{CLOsm}\right)$, filtration fraction $(\mathrm{FF}=$ $\mathrm{CLCr} / \mathrm{CIPAH}$ ), sodium fractional excretion ( $\mathrm{FENa}=$ CLNa.100/CLCr), potassium fractional excretion (FEK = CLK.100/CLCr) and vasopressin plasma levels (VPC).

Parameters were evaluated in 4 moments. Each moment lasted 15 minutes and blood collection and hemodynamic variables evaluation were performed at half this interval. Bladder was emptied in the beginning and end of each moment and urine was collected. Studied moments were:

M1 (control): immediately after the 30 -minute stabilization period.

M2: after initial injection, in 10 minutes, of $20 \mathrm{~mL}$ of the solution in study, coinciding with the beginning of continuous injection of the solution in study, in one hour.

M3: 30 minutes after M2, coinciding the end of this moment with the end of the injection of the solution in study.

M4: 30 minutes after M3.

To measure vasopressin plasma levels (VPL), $5 \mathrm{~mL}$ of venous blood were collected in tubes with heparin, which were centrifuged at $4^{\circ} \mathrm{C}$ for plasma separation. Radioimmunoassay was the technique of choice (kit DSL-1800 Arginine Vasopressin Radioimmunoassay, Texas) with readings by Perkim-Elmer Cobra II Gamma Counter, model E5005 (USA) device. Vasopressin plasma levels were expressed in picograms per $\mathrm{mL}$ of plasma.

Profile analysis, followed by Tukey's method for multiple comparisons were used for variables with normal distribution and homogeneity of variances. For variables without normal distribution or homogeneity of variances, Friedman's test was used to compare moments and Kruskal-Wallis test was used to compare groups, followed by multiple comparisons test. Analysis of variance was used to evaluate demographics. Fisher Exact test for frequency analysis was used for gender. Significance level was $5 \%$.

\section{RESULTS}

Groups were homogeneous in weight, length, BSA and gender distribution $(p>0.05)$ (Table I). 
There were no significant differences in MBP and DBP among groups and in different moments within each group $(p>0.05)$ (Table II).

Table I - Weight, Length, Body Surface Area (BSA) and Gender distribution in Each Studied Group

\begin{tabular}{lcccc}
\hline Parameters & $\begin{array}{c}\mathrm{G} 1 \\
(\mathrm{n}=12)\end{array}$ & $\begin{array}{c}\mathrm{G} 2 \\
\mathrm{n}=12)\end{array}$ & $\begin{array}{c}\mathrm{G} 3 \\
(\mathrm{n}=12)\end{array}$ & Statistics \\
\hline Weight $(\mathrm{kg}){ }^{*}$ & $24.3 \pm 4.2$ & $21.9 \pm 3.9$ & $23.9 \pm 3.7$ & $\mathrm{p}>0.05$ \\
Length $(\mathrm{cm}){ }^{*}$ & $114.7 \pm 6.9$ & $107.3 \pm 8.0$ & $114.8 \pm 10.1$ & $\mathrm{p}>0.05$ \\
$\mathrm{BSA}\left(\mathrm{m}^{2}\right)^{*}$ & $0.90 \pm 0.09$ & $0.85 \pm 0.09$ & $0.86 \pm 0.11$ & $\mathrm{p}>0.05$ \\
Gender & & & & \\
\multicolumn{1}{c}{ Males } & 9 & 10 & 12 & \\
Females & 3 & 2 & 0 & $\mathrm{p}>0.05$ \\
\hline
\end{tabular}

*Values expressed in Mean \pm SD

HR was lower for $G 2$ and $G 3$ as compared to $G 1$ in $M 2$, M3 and M4 ( $p<0.05)$. For $G 2, H R$ has decreased in M2 $(p<0.05)$, returning to baseline values in M3 and M4. For G3, HR has decreased in $\mathrm{M} 2$ and was kept below baseline values in $\mathrm{M} 3$ and M4 $(p<0.05)$. G1 had progressive HR increase $(p<0.05)$ (Table II).
$\mathrm{Cl}$ in M4 was lower for $\mathrm{G} 2$ and $\mathrm{G} 3$ as compared to $\mathrm{G} 1$; and for $\mathrm{G} 3$ as compared to $\mathrm{G} 2(\mathrm{p}<0.05)$. There has been progressive $\mathrm{Cl}$ increase for $\mathrm{G} 1$ throughout the moments $(p<0.05)$ (Table II).

SVRI was lower for G1 in M2, M3 and M4 as compared to G2 and $G 3(p<0.05)$. There has been SVRI decrease for $G 1$ in $M 2, M 3$ and M4 as compared to M1 ( $<<0.05)$ (Table II). UO was lower for $\mathrm{G} 1$ in M4 ( $p<0.05)$. For $G 3$, UO was lower in $M 2$ as compared to M3 ( $<0.05)$ (Figure 1).

FF was lower for $\mathrm{G} 2$ as compared to $\mathrm{G} 1$ in $\mathrm{M} 1(\mathrm{p}<0.05)$. For G2, it was higher in M4 as compared to M1 $(p<0.05)$ (Table III).

OsmU was not significantly different among groups. For G2 and $\mathrm{G} 3$ it was lower in M3 and M4 $(p<0.05)$ (Table IV). $\mathrm{CLH}_{2} \mathrm{O}$ was not significantly different among groups. For $\mathrm{G} 3$ it was higher in M2, M3 and M4 as compared to M1 $(p<0.05)$ (Figure 2).

Vasopressin plasma level was lower for $\mathrm{G} 3$ as compared to G1 and $G 2$, and for $G 2$ as compared to $G 1$, in $M 2$ and $M 4$ $(p<0.05)$. For $G 3$, it was higher in $M 1(p<0.05)$ (Figure 3$)$.

RBF, GFR, RVR (Table III), Ht, OsmP, CLOsm, FENa and FEK (Table IV) were not significantly different among groups and in different moments within each group $(p>0.05)$.

Table II - Heart Rate (HR), Mean Blood Pressure (MBP), Mean Diastolic Blood Pressure (DBP), Cardiac Index (Cl) and Systemic Vascular Resistance Index (SVRI) in Studied Moments and Groups

\begin{tabular}{|c|c|c|c|c|}
\hline \multirow{2}{*}{ Variables } & \multicolumn{4}{|c|}{ Moments } \\
\hline & M1 & M2 & M3 & M4 \\
\hline \multicolumn{5}{|c|}{$\mathrm{HR}^{1}\left(\right.$ beat. $\left.\min ^{-1}\right)$} \\
\hline $\mathrm{G} 1$ & $93[85 ; 106] \mathrm{A}^{3} \mathrm{c}^{4}$ & $105[100 ; 130] \mathrm{Ab}$ & $133[110 ; 155] \mathrm{Aa}$ & $128[110 ; 146] \mathrm{Aa}$ \\
\hline $\mathrm{G} 2$ & $110[97 ; 137] \mathrm{Aa}$ & $69[62 ; 114] \mathrm{Bb}$ & $102[80 ; 120] \mathrm{Ba}$ & $108[99 ; 124] \mathrm{Ba}$ \\
\hline G3 & $103[93 ; 124] \mathrm{Aa}$ & 78 [69; 99] Bb & $81[71 ; 104] \mathrm{Bb}$ & $91[85 ; 112] \mathrm{Bb}$ \\
\hline \multicolumn{5}{|c|}{$\mathrm{MBP}^{2.5}(\mathrm{mmHg})$} \\
\hline $\mathrm{G} 1$ & $91.8 \pm 16.0$ & $91.1 \pm 16.6$ & $90.1 \pm 15.4$ & $94.3 \pm 16.2$ \\
\hline $\mathrm{G} 2$ & $95.3 \pm 13.8$ & $91.1 \pm 10.0$ & $94.9 \pm 7.9$ & $99.8 \pm 9.4$ \\
\hline G3 & $97.5 \pm 19.7$ & $89.5 \pm 16.3$ & $91.6 \pm 14.4$ & $97.7 \pm 15.8$ \\
\hline \multicolumn{5}{|c|}{$\mathrm{DBP}^{1.5}(\mathrm{mmHg})$} \\
\hline $\mathrm{G} 1$ & $3.5[1.5 ; 5.0]$ & $3.0[2.5 ; 4.5]$ & $3.0[2.5 ; 4.0]$ & $3.5[2.5 ; 4.0]$ \\
\hline $\mathrm{G} 2$ & $2.5[1.0 ; 4.0]$ & $5.0[3.0 ; 7.0]$ & $5.0[3.0 ; 7.0]$ & $4.0[3.0 ; 4.5]$ \\
\hline G3 & $2.0[1.0 ; 4.0]$ & $3.0[1.5 ; 5.5]$ & $3.0[2.0 ; 5.5]$ & $2.5[1.5 ; 5.0]$ \\
\hline \multicolumn{5}{|c|}{$\mathrm{Cl}^{1}\left(\mathrm{~L} \cdot \mathrm{min}^{-1} \cdot \mathrm{m}^{-2}\right)$} \\
\hline G1 & $3.4[3.0 ; 4.3] \mathrm{Ac}$ & $3.9[3.4 ; 4.8] \mathrm{Ab}$ & $4.9[4.2 ; 5.5] \mathrm{Aa}$ & $4.5[3.8 ; 5.2] \mathrm{Aa}$ \\
\hline $\mathrm{G} 2$ & $3.3[2.9 ; 4.6] \mathrm{Aa}$ & $2.6[2.2 ; 3.8] \mathrm{Aa}$ & $3.6[3.6 ; 2.9] \mathrm{Ba}$ & $3.4[2.6 ; 3.8] \mathrm{Ba}$ \\
\hline G3 & $3.5[2.9 ; 4.3] \mathrm{Aa}$ & $2.6[2.3 ; 3.3] \mathrm{Aa}$ & $3.2[2.5 ; 3.5] \mathrm{Ca}$ & $3.1[2.3 ; 3.2] \mathrm{Ca}$ \\
\hline \multicolumn{5}{|c|}{$\mathrm{SVRI}^{1}\left(\right.$ dina.s. $\left.\mathrm{cm}^{-5} \cdot \mathrm{m}^{-2}\right)$} \\
\hline G1 & $1862[1669 ; 2460] \mathrm{Aa}$ & $1594[1481 ; 2078] \mathrm{Bb}$ & $1342[1203 ; 1703] \mathrm{Bd}$ & $1463[1388 ; 1967] \mathrm{Bc}$ \\
\hline $\mathrm{G} 2$ & 2127 [1770; 2536] Aa & $2490[1709 ; 3267] \mathrm{Aa}$ & $2069[1721 ; 2478] \mathrm{Aa}$ & $2284[1990 ; 2887] \mathrm{Aa}$ \\
\hline G3 & $2278[1827 ; 2457] \mathrm{Aa}$ & 2414 [2034; 2923] Aa & $2200[1855 ; 2831] \mathrm{Aa}$ & $2428[2234 ; 3088] \mathrm{Aa}$ \\
\hline
\end{tabular}

${ }^{1}$ Median, $1^{\text {st }}$ and $3^{\text {rd }}$ quartiles; ${ }^{2}$ Mean $\pm S D ;{ }^{3}$ Groups within each moment followed by the same upper case letter are not statistically different ( $\left.\mathrm{p}>0.05\right) ;{ }^{4}$ Moments within each group followed by the same lower case letter are not statistically different ( $p>0.05) ;{ }^{5} p>0.05$ (among groups within each moment, and among moments within each group); ${ }^{3.4}$ Observation as to values: $A>B>C ; a>b>c>d$ 
Table III - Renal Blood Flow (RBF), Glomerular Filtration Rate (GFR), Renal Vascular Resistance (RVR) and Filtration Fraction (FF) in Studied Moments and Groups

\begin{tabular}{|c|c|c|c|c|}
\hline \multirow{2}{*}{ Variables } & \multicolumn{4}{|c|}{ Moments } \\
\hline & M1 & M2 & M3 & M4 \\
\hline \multicolumn{5}{|c|}{$\mathrm{RBF}^{2}\left(\mathrm{~mL} \cdot \mathrm{min}^{-1} \cdot \mathrm{kg}^{-1}\right)$} \\
\hline $\mathrm{G} 1$ & $16.1 \pm 5.2$ & $20.9 \pm 7.3$ & $18.8 \pm 5.4$ & $19.1 \pm 6.5$ \\
\hline G2 & $24.5 \pm 8.3$ & $20.0 \pm 6.4$ & $20.1 \pm 4.4$ & $21.1 \pm 5.6$ \\
\hline G3 & $19.1 \pm 10.0$ & $17.9 \pm 10.3$ & $19.5 \pm 11.4$ & $17.8 \pm 9.5$ \\
\hline \multicolumn{5}{|c|}{$\mathrm{GFR}^{1.5}\left(\mathrm{~mL} \cdot \mathrm{min}^{-1} \cdot \mathrm{kg}^{-1}\right)$} \\
\hline $\mathrm{G} 1$ & $3.4[2.5 ; 4.3]$ & $3.9[3.1 ; 5.0]$ & $3.7[3.2 ; 4.7]$ & $3.5[3.0 ; 4.0]$ \\
\hline G2 & $3.7[2.8 ; 4.4]$ & $4.1[3.8 ; 4.6]$ & $3.9[3.2 ; 4.4]$ & $4.0[3.7 ; 4.8]$ \\
\hline G3 & $3.4[2.6 ; 4.8]$ & $3.5[2.9 ; 4.8]$ & $3.7[3.4 ; 4.5]$ & $3.8[3.4 ; 4.3]$ \\
\hline \multicolumn{5}{|c|}{$\mathrm{RVR}^{1.5}\left(\mathrm{mmHg} \cdot \mathrm{mL}^{-1} \cdot \mathrm{min}^{-1}\right)$} \\
\hline G1 & $0.25[0.19 ; 0.32]$ & $0.19[0.14 ; 0.25]$ & $0.18[0.16 ; 0.24]$ & $0.20[0.16 ; 0.27]$ \\
\hline $\mathrm{G} 2$ & $0.17[0.15 ; 0.22]$ & $0.23[0.19 ; 0.26]$ & $0.24[0.18 ; 0.28]$ & $0.26[0.20 ; 0.30]$ \\
\hline G3 & $0.24[0.17 ; 0.32]$ & $0.24[0.14 ; 0.34]$ & $0.23[0.16 ; 0.30]$ & $0.26[0.18 ; 0.36]$ \\
\hline \multicolumn{5}{|l|}{$\mathrm{FF}^{2}$} \\
\hline G1 & $0.38 \pm 0.09 \mathrm{a} 3 \mathrm{~A} 4$ & $0.34 \pm 0.09 \mathrm{aA}$ & $0.36 \pm 0.09 \mathrm{aA}$ & $0.35 \pm 0.07 \mathrm{aA}$ \\
\hline G2 & $0.27 \pm 0.09 \mathrm{bB}$ & $0.33 \pm 0.06 \mathrm{abA}$ & $0.34 \pm 0.10 \mathrm{abA}$ & $0.35 \pm 0.07 \mathrm{aA}$ \\
\hline G3 & $0.37 \pm 0.09 \mathrm{aAB}$ & $0.38 \pm 0.09 \mathrm{aA}$ & $0.36 \pm 0.09 \mathrm{aA}$ & $0.41 \pm 0.07 \mathrm{aA}$ \\
\hline
\end{tabular}

Table IV - Hematocrit (Ht), Urinary Osmolality (OsmU), Plasma Osmolality (OsmP), Osmolar Clearance (CLOsm), Sodium Fractional Excretion (FENa) and Potassium Fractional Excretion (FEK) in Studied Moments and Groups

\begin{tabular}{|c|c|c|c|c|}
\hline \multirow[t]{2}{*}{ Variables } & \multicolumn{4}{|c|}{ Moments } \\
\hline & M1 & M2 & M3 & M4 \\
\hline \multicolumn{5}{|l|}{$\mathrm{Ht}^{2}(\%)$} \\
\hline G1 & $39.5[36.0 ; 43.0]$ & $40.0[36.5 ; 44.0]$ & $41.0[37.0 ; 43.5]$ & $41.0[37.0 ; 42.5]$ \\
\hline G2 & $41.0[38.5 ; 44.5]$ & $42.0[36.0 ; 46.0]$ & $41.5[39.0 ; 46.5]$ & $43.5[40.0 ; 46.0]$ \\
\hline G3 & $39.5[36.5 ; 42.0]$ & $41.0[35.5 ; 44.0]$ & $41.5[36.0 ; 44.0]$ & $41.5[36.0 ; 45.0]$ \\
\hline \multicolumn{5}{|c|}{ OsmU (mOsm.kg H $\mathrm{O}^{-1}$ ) } \\
\hline G2 & $754[561 ; 994]$ a & 641 [536; 868] a & $358[302 ; 639] \mathrm{b}$ & $306[234 ; 655] \mathrm{b}$ \\
\hline G3 & 925 [795; 1346] a & 795 [605; 986] a & $452[313 ; 558] \mathrm{b}$ & $344[282 ; 604] \mathrm{A}$ \\
\hline \multicolumn{5}{|c|}{$\mathrm{OsmP}^{2}$ (mOsm.kg H $\mathrm{O}^{-1}$ ) } \\
\hline G1 & $288[278 ; 295]$ & $284[278 ; 288]$ & 288 [287; 290] & $288[285 ; 293]$ \\
\hline G2 & 290 [286; 295] & $293[284 ; 296]$ & 288 [284; 292] & $290[283 ; 295]$ \\
\hline G3 & $281[252 ; 290]$ & $285[238 ; 295]$ & 286 [269; 290] & $287[258 ; 293]$ \\
\hline G1 & $1.9[1.2 ; 2.2]$ & $2.2[1.6 ; 2.7]$ & $1.9[1.6 ; 2.7]$ & $1.8[1.2 ; 2.0]$ \\
\hline G2 & $1.9[1.1 ; 2.9]$ & $1.4[1.2 ; 2.5]$ & $1.8[1.4 ; 3.2]$ & $1.7[1.2 ; 3.3]$ \\
\hline G3 & $2.1[1.6 ; 3.0]$ & $1.6[1.1 ; 1.9]$ & $1.9[1.5 ; 4.0]$ & $1.7[1.4 ; 2.3]$ \\
\hline \multicolumn{5}{|l|}{$\mathrm{FENa}^{2}(\%)$} \\
\hline G1 & $0.38[0.23 ; 0.77]$ & $0.89[0.44 ; 1.07]$ & $0.94[0.25 ; 1.21]$ & $0.42[0.20 ; 0.64]$ \\
\hline G2 & $0.57[0.31 ; 1.08]$ & $0.55[0.37 ; 0.83]$ & $1.02[0.75 ; 1.28]$ & $0.59[0.40 ; 0.81]$ \\
\hline G3 & $0.40[0.23 ; 0.96]$ & $0.37[0.21 ; 1.05]$ & $0.68[0.42 ; 1.09]$ & $0.60[0.31 ; 0.99]$ \\
\hline \multicolumn{5}{|l|}{$\mathrm{FEK}^{2}(\%)$} \\
\hline G1 & $12.0[7.9 ; 24.7]$ & $16.7[12.1 ; 29.2]$ & $24.7[14.9 ; 31.2]$ & $21.0[12.4 ; 26.2]$ \\
\hline G2 & $21.8[11.1 ; 31.2]$ & 15.7 [11.0; 26.8] & $31.1[23.4 ; 37.6]$ & $20.3[15.7 ; 28.8]$ \\
\hline G3 & $20.0[12.7 ; 29.2]$ & $15.0[5.7 ; 21.7]$ & $25.9[23.0 ; 28.0]$ & $20.2[14.3 ; 25.3]$ \\
\hline
\end{tabular}

Values in median, $1^{\text {st }}$ and $3^{\text {rd }}$ quartiles; ${ }^{1}$ Moments within each group followed by the same lower case letter are not statistically different $(p>0.05) ;{ }^{2} p>0.05$ (among groups within each moment, and among moments within each group); ${ }^{1}$ Observation as to values: $a>b$ 


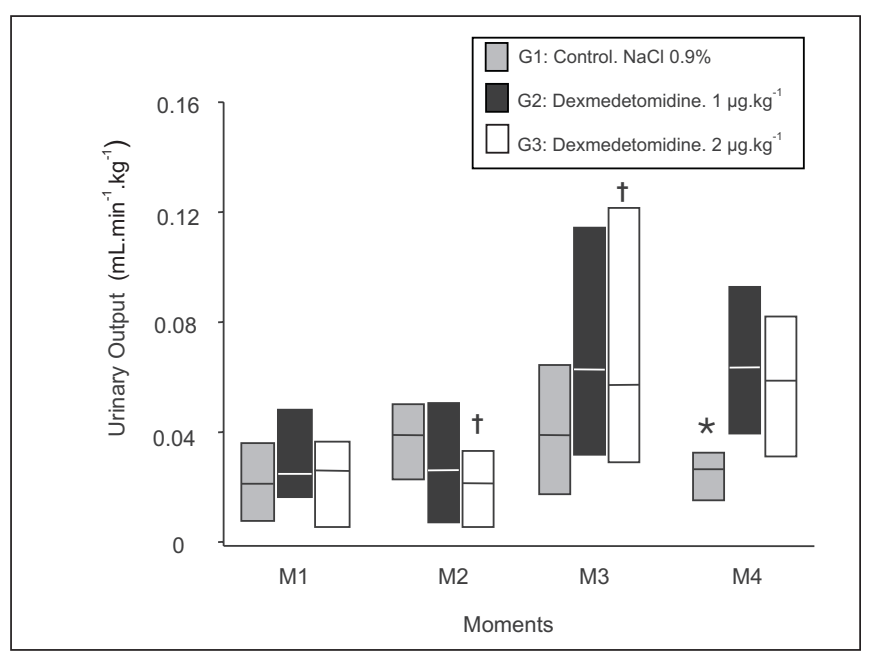

Figure 1 - Urinary Output. Median, $1^{\text {st }}$ and $3^{\text {rd }}$ Quartiles of Values Obtained in Studied Groups and Moments

* $\mathrm{M} 4: \mathrm{G} 1<(\mathrm{G} 2=\mathrm{G} 3)(\mathrm{p}<0.05)$

+ G3: M2<M3 $(p<0.05)$

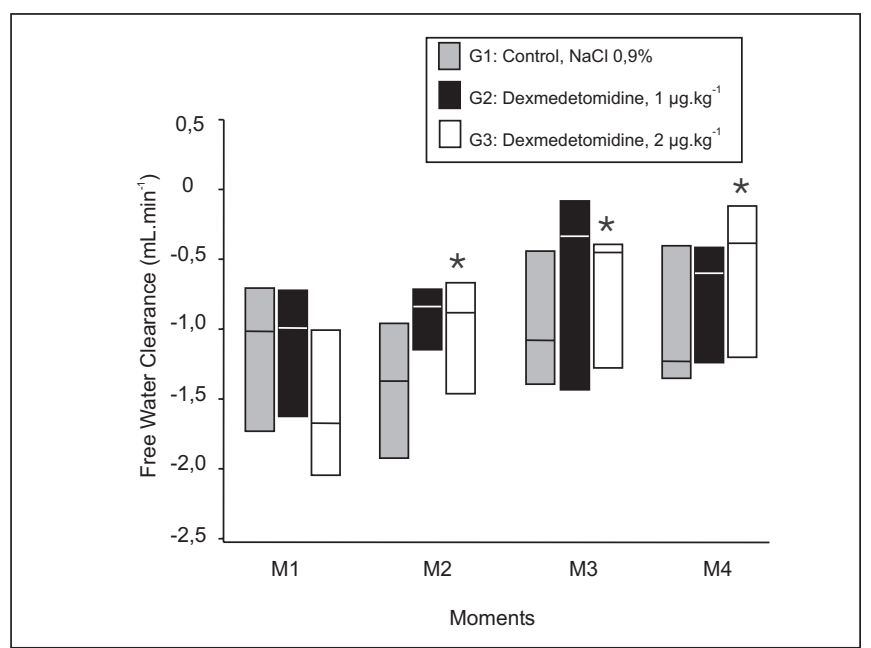

Figure 2 - Free Water Clearance. Median, $1^{\text {st }}$ and $3^{\text {rd }}$ Quartiles of Values Obtained in Studied Groups and Moments

${ }^{*} \mathrm{G} 3: \mathrm{M} 1<(\mathrm{M} 2=\mathrm{M} 3=\mathrm{M} 4)(\mathrm{p}<0.05)$

\section{DISCUSSION}

Glomerular filtration rate is a predominantly hemodynamic mechanism and the renal intratubular dynamics suffers hemodynamic and hormonal influences. Some hormones, such as vasopressin, suffer cardiovascular interferences to control their secretion ${ }^{11}$. Our experiment has observed that urinary changes promoted by dexmedetomidine were not followed by significant hemodynamic changes. Similar to other studies $^{6-10,12}$, dexmedetomidine has dose-dependently decreased $\mathrm{HR}$ and has maintained better hemodynamic stability as compared to control, and the $\mathrm{Cl}$ was maintained stable throughout the experiment in both groups receiving the drug.

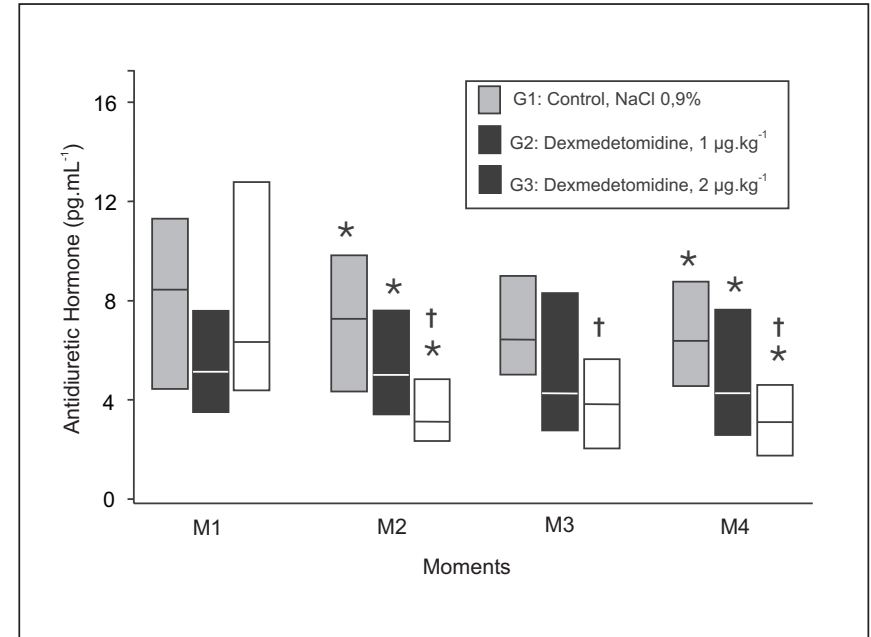

Figure 3 - Vasopressin Plasma Concentration. Median, $1^{\text {st }}$ and $3^{\text {rd }}$ Quartiles of Values Obtained in Studied Groups and Moments * M2 e M4: G1>G2>G3 (p>0.05)

† G3: $M 1>(M 2=M 3=M 4)(p<0.05)$

Alpha ${ }_{2}$-adrenergic agonists increase urinary output ${ }^{12-15}$. This may be secondary to hemodynamic changes ${ }^{16}$, to vasopressin secretion inhibition ${ }^{17}$ or to its decreased tubular action $^{18}$.

Alpha $_{2}$-adrenergic receptors have already been identified in several renal areas of different animals ${ }^{19-25}$. In rats, the activation of these receptors promotes intracellular cAMP inhibition ${ }^{26,27}$, being this one mechanism responsible for vasopressin action inhibition in the collecting duct. In other animals, such as dogs, some investigators have not detected such intracellular cAMP inhibition ${ }^{16}$, suggesting a different mechanism involved in inhibiting collecting duct water absorption by $\alpha_{2}$ - adrenergic agonists. Reid et al. ${ }^{17}$ have reported that very high clonidine doses, well above those normally used, inhibit vasopressin secretion promoting increased diuresis.

Our study has observed that dexmedetomidine has determined urinary output increase, promoting minor parallel hemodynamic changes. Increased diuresis was followed by decreased urinary osmolality and increased free water clearance. There were no changes in RVR, RBF, GFR, CLOsm, FE $\mathrm{Na}$ and $\mathrm{FEK}$, confirming that increased diuresis was secondary to the impairment of water absorption in the collecting duct and not secondary to glomerular filtration increase. This acqueous diuresis was probably secondary to vasopressin secretion inhibition.

Humphrey et al. ${ }^{28}$ have associated vasopressin secretion inhibition after clonidine injection to increased MBP. Other possible mechanisms would be no secretion of this hormone due to increased central venous pressure ${ }^{29}$ or direct blockade of supraoptic neurosecretory cells ${ }^{30}$.

In our experiment, dexmedetomidine has dose-dependently inhibited vasopressin secretion with significant MBP or DBP changes, suggesting direct central inhibition of the drug. In a previous study, Kimura et al. ${ }^{31}$ have observed decreased 
vasopressin plasma levels after low clonidine dose injected in the lateral ventricle of dogs, showing that $\alpha_{2}$-agonists may promote central vasopressin secretion blockade.

We have observed during control moment that vasopressin plasma levels were close to dogs' physiological values ( 0 to 5 $\left.\mathrm{pg} \cdot \mathrm{mL}^{-1}\right)^{32}$, possibly being result of hydration during surgical preparation and of balanced anesthetic technique with opioids during induction ${ }^{33}$. Other investigators have observed very high vasopressin plasma levels after anesthetic-surgical procedures in dogs ${ }^{34}$. There has been decreased vasopressin plasma levels, increased urinary output and low osmolality urine after dexmedetomidine administration in doses close to those used in Anesthesiology ${ }^{35}$. There were no hemodynamic changes which could justify decreased vasopressin secretion and higher urine production. In experimental and clinical trials, blockade of endocrine-metabolic response to anesthetic-surgical trauma associated to clonidine renal effects could prevent or decrease renal injuries secondary to renal ischemia ${ }^{36,37}$

So, we have concluded that low dexmedetomidine doses in dogs result in aqueous diuresis secondary to central vasopressin secretion inhibition. Based on these actions and on clonidine trials resulting in renal protection against ischemia, it is suggested that dexmedetomidine plays a potential role in protecting kidneys against ischemic events. Experimental renal ischemia models, as well as clinical trials in patients susceptible to renal ischemia, are needed to confirm this hypothesis.

\section{REFERÊNCIAS - REFERENCES}

01. Godet G, Fleron MH, Vicaut E et al - Risk factors for acute postoperative renal failure in thoracic or thoracoabdominal aortic surgery: a prospective study. Anesth Analg, 1997;85:1227-1232.

02. Mangano CM, Diamondstone LS, Ramsay JG et al - Renal dysfunction after myocardial revascularization: risk factors, adverse outcomes and hospital resource utilization. The Multicenter study of Perioperative Ischemia Research Group. Ann Intern Med, 1998;128:194-203.

03. Conlon PJ, Stafford-Smith M, White WD et al - Acute renal failure following cardiac surgery. Nephrol Dial Transplant, 1999;14:1158-1162.

04. Swaminathan M, Stafford-Smith M - Renal dysfunction after vascular surgery. Curr Opin Anaesthesiol, 2003;16:45-51.

05. Lema G, Canessa R, Urzua J - Renal preservation in cardiac surgery. Curr Opin Anesthesiol, 1998;11:9-13.

06. Scheinin M, Kallio A, Koulu M et al - Sedative and cardiovascular effects of medetomidine, a novel seletive alpha2-adrenoceptor agonist, in healthy volunteers. Br J Clin Pharmacol, 1987;24: 443-451.

07. Kallio A, Scheinin M, Koulu M et al - Effects of dexmedetomidine, a seletive alpha2-adrenoceptor agonist, on hemodynamic control mechanisms. Clin Pharmacol Ther, 1989;46:33-42.

08 . Aho M, Scheinin M, Lehtinen AM et al - Intramusculary administered dexmedetomidine attenuates hemodynamic and stress hormone responses to gynecologic laparoscopy. Anesth Analg, 1992; 75:932-939.
09. Talke P, Chen R, Thomas B et al - The hemodynamic and adrenergic effects of perioperative dexmedetomidine infusion after vascular surgery. Anesth Analg, 2000;90:834-839.

10. Jalonen J, Hynynen M, Kuitunen A et al - Dexmedetomidine as an anesthetic adjunct in coronary artery bypass grafting. Anesthesiology, 1997;86:331-345.

11. Aires MM - Fisiologia Renal, em: Aires MM - Fisiologia. Rio de Janeiro: Guanabara Koogan, 1999:473-488.

12. Maze M, Tranquilli W - Alpha-2 adrenoceptor agonists: defining the role in clinical anesthesia. Anesthesiology, 1991;74: 581-605.

13. Hamaya Y, Nishikawa T, Dohi S - Diuretic effect of clonidine during isoflurane, nitrous oxide, and oxygen anesthesia. Anesthesiology, 1994;81:811-819.

14. Evans RG, Shweta A, Malpas SC et al - Renal effects of rilmenidine in volume-loaded anaesthetized dogs. Clin Exp Pharmacol Physiol, 1997;24:64-67.

15. Cabral AD, Kapusta DR, Kenigs VA et al - Central alpha2-receptor mechanisms contribute to enhanced renal responses during ketamine-xylazine anesthesia. Am J Physiol, 1998;275: R1867-R1874.

16. Brooks DP, Edwards RM, Depalma PD et al - The water diuretic effect of the alpha-2 adrenoceptor agonist, AGN 190851, is species-dependent. J Pharmacol Exp Ther, 1991;259: $1277-1282$

17. Reid IA, Nolan PL, Wolf JA et al - Suppression of vasopressin secretion by clonidine: effect of alpha-adrenoceptor antagonists. Endocrinology, 1979;104:1403-1406.

18. Rouch AJ, Kudo LH, Hebert C - Dexmedetomidine inhibits osmotic water permeability in the rat cortical collecting duct. $J$ Pharmacol Exp Ther, 1997;281:62-69.

19. Schmitz JM, Graham RM, Sagalowsky A et al - Renal alpha-1 and alpha-2 adrenergic receptors: biochemical and pharmacological correlations. J Pharmacol Exp Ther, 1981;219: 400-406.

20. Michel MC, Brodde OE, Schnepel B et al - $[3 \mathrm{H}]$-idazoxan and some other alpha2-adrenergic drugs also bind with affinity to a nonadrenergic site. Mol Pharmacol, 1989;35:324-330.

21. Calianos T 2nd, Muntz KH - Autoradiographic quantification of adrenergic receptors in rat kidney. Kidney Int, 1990;38:39-46.

22. Stanko CK, Vandel MI, Bose R et al - Characterization of alpha2-adrenoceptors in the rat: proximal tubule, renal membrane and whole kidney studies. Eur J Pharmacol, 1990;175: 13-20.

23. Clarke D, Garg LC - Alpha-2 adrenergic receptors in inner medullary collecting duct cells of the rabbit kidney. J Pharmacol Exp Ther, 1993;264:879-888.

24. Mohuczy-Dominiak D, Garg LC - Alpha-2 adrenoceptors in medullary thick ascending limbs of the rabbit kidney. J Pharmacol Exp Ther, 1993;266:279-287.

25. Evans RG, Haynes JM - Characterization of binding sites for $[3 \mathrm{H}]$-idazoxan, [3H]-P-aminoclonidine and [3H]-rauwolscine in the kidney of the dog. Clin Exp Pharmacol Physiol, 1994;21: 649-658.

26. Chabardes D, Montegut M, Imbert-Teboul M et al - Inhibition of alpha2-adrenergic agonists on AVP-induced cAMP accumulation in isolated collecting tubules of the rat kidney. Mol Cell Endocrinol, 1984;37:263-275.

27. Umemura S, Marver D, Smyth DD et al - Alpha2-adrenoceptors and cellular CAMP levels in single nephron segments from the rat. Am J Physiol, 1985;249:F28-F33.

28. Humphreys MH, Reid IA - Suppression of antidiuretic hormone secretion by clonidine in the anesthetized dog. Kidney Int, $1975 ; 7: 405-412$. 
29. Barr JG, Kauker ML - Renal tubular site and mechanism of clonidine-induced diuresis in rats: clearance and micropuncture studies. J Pharmacol Exp Ther, 1979;209:389-395.

30. Barker JL, Crayton JW, Nicoll RA - Noradrenaline and acetylcholine responses of supraoptic neurosecretory cells. J Physiol, 1971;218:19-32.

31. Kimura T, Share L, Wang BC et al - The role of central adrenoreceptors in the control of vasopressin release and blood pressure. Endocrinology, 1981;108:1829-1836.

32. Cowley AW Jr - Vasopressin and cardiovascular regulation. Int Rev Physiol, 1982:26:189-242.

33. Leander JD, Zerbe RL, Hart JC - Diuresis and supresion of vasopressin by kappa opioids: comparison with mu and delta opioids and clonidine. J Pharmacol Exp Ther, 1985;234: 463-469.

34. Reid IA, Ahn JN, Trinh T et al - Mechanism of suppression of vasopressin and adrenocorticotropic hormone secretion by clonidine in anesthetized dogs. J Pharmacol Exp Ther, 1984;229:1-8.

35. Villela NR, Nascimento Jr P - Uso de dexmedetomidina em Anestesiologia. Rev Bras Anestesiol, 2003;53:97-113.

36. Solez K, Ideura T, Silvia CB et al - Clonidine after renal ischemia to lessen acute renal failure and microvascular damage. Kidney Int, 1980;18:309-322.

37. Kulka P, Tryba M, Zenz M - Preoperative alpha2-adrenergic receptor agonists prevent the deterioration of renal function after cardiac surgery: results of a randomized, controlled trial. Crit Care Med, 1996;24:947-952.

\section{RESUMEN}

Villela NR, Nascimento Jr P, Carvalho LR, Teixeira A - Efectos de la Dexmedetomidina sobre el Sistema Renal y sobre la Concentración Plasmática de la Hormona Antidiurética. Estudio Experimental en Perros

JUSTIFICATIVA Y OBJETIVOS: La insuficiencia renal aguda peri-operatoria es responsable de la elevada tasa de morbidad y mortalidad. Los fármacos $\alpha_{2}$-agonistas aumentan el débito urinario y promueven buena estabilidad hemodinámica en ese período. El objetivo de esta pesquisa fue estudiar los efectos renales y sobre la concentración plasmática de la hormona antidiurética (HAD) provocados por la dexmedetomidina en un perro anestesiado.

MÉTODO: Treinta seis perros adultos, anestesiados con propofol, fentanil e isoflurano, fueron divididos eventualmente en tres grupos que recibieron, de modo encubierto: G1 inyección de $20 \mathrm{~mL}$ de solución de cloruro de sodio a 0,9\%, en 10 minutos, seguida de inyección de $20 \mathrm{~mL}$ de la misma solución en una hora; G2 - inyección de 20 mL de solución de cloruro de sodio a $0,9 \%$ conteniendo dexmedetomidina $\left(1 \mu \mathrm{g} \cdot \mathrm{kg}^{-1}\right)$, en 10 minutos, seguida de inyección de $20 \mathrm{~mL}$ de la misma solución, con la misma dosis de dexmedetomidina $\left(1 \mu \mathrm{g} \cdot \mathrm{kg}^{-1}\right)$, en una hora y $\mathrm{G} 3$ - inyección de $20 \mathrm{~mL}$ de solución de cloruro de sodio a $0,9 \%$ conteniendo dexmedetomidina $\left(2 \mu \mathrm{g} . \mathrm{kg}^{-1}\right)$ en 10 minutos, seguida de inyección de $20 \mathrm{~mL}$ de la misma solución, con la misma dosis de dexmedetomidina $\left(2 \mu \mathrm{g} \cdot \mathrm{kg}^{-1}\right)$, en una hora. Las variables renales, hemodinámicas y la concentración plasmática del HAD fueron estudiadas en cuatro momentos: M1 (control) - inmediatamente después del período de estabilización; M2 - después de la inyección inicial de $20 \mathrm{~mL}$ de la solución en estudio, en 10 minutos, coincidiendo con el inicio de la inyección de la misma solución, en una hora; M3 - 30 minutos después de M2 y M4 - 30 minutos después de M3.

RESULTADOS: La dexmedetomidina redujo la frecuencia cardiaca y promovió estabilidad hemodinámica, manteniendo constante el débito cardíaco. Hubo elevación del débito urinario en el G2 y G3, en comparación con el G1.La osmolalidad urinaria en el G2 y G3 fue menor en el M3 y M4 con relación al M1 y M2. La depuración de agua libre aumentó en el G3. La concentración plasmática del HAD diminuyó en el G3, presentando valores más bajos que los observados en el G1 y G2 en M2 y M4.

CONCLUSIONES: Los perros anestesiados con bajas dosis de dexmedetomidina promueven diuresis hídrica por inhibir la secreción de la hormona antidiurética, habiendo potencial para la protección renal en eventos isquémicos. 